\title{
G9A performs important roles in the progression of breast cancer through upregulating its targets
}

\author{
WENHUA JIANG $^{1^{*}}$, PENGFEI LIU ${ }^{2 *}$ and XIAODONG LI ${ }^{1}$ \\ ${ }^{1}$ Department of Radiotherapy, Second Hospital of Tianjin Medical University, Tianjin 300211; ${ }^{2}$ Department of Lymphoma, \\ Sino-US Center of Lymphoma and Leukemia, Tianjin Medical University Cancer Institute and Hospital, Tianjin's National \\ Clinical Research Center for Cancer, Key Laboratory of Cancer Prevention and Therapy, Tianjin 300060, P.R. China
}

Received April 26, 2016; Accepted February 13, 2017

DOI: $10.3892 / \mathrm{ol} .2017 .5977$

\begin{abstract}
Breast cancer (BC) is the most common type of malignancy in females worldwide, however, its underlying mechanisms remain poorly understood. The present study aimed to investigate the mechanisms behind the development and progression of $\mathrm{BC}$ and identify potential biomarkers for it. The chromatin immunoprecipitation-DNA sequencing (ChIP-Seq) dataset GSM1642516 and gene expression dataset GSE34925 were downloaded from the Gene Expression Omnibus database. Affy and oligo packages were used for the background correction and normalization of the gene expression dataset. Based on Limma package and the criteria of a fold change $>1.41$ or $<0.71$, and a false discovery rate adjusted P-value $<0.05$, differentially-expressed genes (DEGs) in euchromatic histone lysine methyltransferase $2(G 9 A)$ -knockout (KO) breast samples compared with control samples were identified. The Database for Annotation, Visualization and Integrated Analysis was used for the functional enrichment analysis of the DEGs. Bowtie 2 and model-based analysis of ChIP-Seq version 14 (macs14) were used for the mapping of raw reads and the identification of G9A binding sites (peaks), respectively. In addition, overlapping genes between the DEGs and genes in the peaks located in -3000 to $3000 \mathrm{bp}$ centered in the transcription start sites (conpeaks) were screened out and microRNAs (miRNAs) believed to regulate those overlaps were identified through the TargetScan database. A total of 217 DEGs were identified in G9A-KO samples, which were mainly involved in the biological processes and pathways associated with the inflammatory response and cancer progression.
\end{abstract}

Correspondence to: Dr Xiaodong Li, Department of Radiotherapy, Second Hospital of Tianjin Medical University, 23 Pingjiang Road, Hexi, Tianjin 300211, P.R. China

E-mail: xiaodonglitj@163.com

*Contributed equally

Key words: breast cancer, chromatin immunoprecipitation-DNA sequencing, gene expression omnibus, database for annotation, visualization and integrated analysis, biomarker
A total of 10,422 peaks, containing 1,210 conpeaks involving 1,138 genes, were identified. Among the 1,138 genes, 15 were overlapped with the DEGs, and 35 miRNAs were identified to regulate those overlaps. Insulin-induced gene 1 was regulated by 9 genes in the miRNA-gene regulation network, which may indicate its importance in the progression of $\mathrm{BC}$. The present study identified potential biomarkers of BC that may be useful in the diagnosis and treatment of patients with the disease.

\section{Introduction}

Breast cancer (BC), is a type of malignancy that poses the greatest threat to female health worldwide, despite its relatively long 5-year survival rate $(\sim 58.75 \%$ between 1972 and 2011 in China) (1). BC is one of the most common types of cancer in Asia, where $39 \%$ of all worldwide cases are diagnosed (2). The treatment and care for patients with BC is a large economic burden in developed and developing countries (3-5). Considering the health and economic effects of $\mathrm{BC}$, numerous studies have been conducted to investigate the pathogenesis, and to identify therapeutic targets and potential factors that may contribute its progression. However, the mechanisms behind the development and progression of $\mathrm{BC}$ remain poorly understood, and additional studies are required.

G9A [also known as euchromatic histone lysine methyltransferase 2 (EHMT2)], encodes a methyltransferase that methylates lysine residues of histone $\mathrm{H} 3$. The dysregulation of $G 9 A$ is associated with numerous abnormal biological processes and the emergence of diseases, including cancer. For example, the downregulation of $G 9 A$ could trigger DNA damage responses and inhibit the progression of colorectal cancer (6). In head and neck squamous cell carcinoma, $G 9 A$ dysregulation has been associated with epithelial-mesenchymal transition (EMT)-mediated metastasis and the maintenance of cancer stem cell-like characteristics (7). Furthermore, it has been identified that $G 9 A$ performs important roles in the transition of acute-to-chronic pain (8). In BC, the aberrant expression of $G 9 A$ could affect the stability of SRY-box 2 (SOX2), a tumor suppressor gene, by inducing its epigenetic silencing (9). Furthermore, a study by Si et al (10) revealed that $G 9 A$ performed important roles in the dysfunction of the reciprocal feedback loop between GATA binding protein 3 and zinc finger E-box binding homeobox 2, and 
that it contributed to the progression of $\mathrm{BC}$. The combined analysis of its genome-wide profiles and the gene expression data following G9A-knockdown (KO) or overexpression in $\mathrm{BC}$ could improve our understanding of its regulatory functions, and has not previously been investigated.

MicroRNAs (miRNAs/miRs) are small non-coding RNA molecules, 22-25 nucleotides in length. miRNAs are formed from the precursor miRNA, a double-stranded hairpin RNA or even the intergenic region, and perform important roles in the regulation of gene expression at the post translation level by silencing mRNA translation or inducing protein degradation (11). Numerous miRNAs have been shown to contribute to the progression of a number of cancer types. For example, the downregulation of $m i R-711$ could inhibit the cellular proliferation of $\mathrm{BC}$, and $m i R-711$ has been shown to be an independent prognostic factor for BC (12). $m i R-27 a / b$ and $m i R-494$ may regulate the expression of tissue factor pathway inhibitor $\alpha$ (TFPI $\alpha)$ in its estrogen-mediated downregulation in $\mathrm{BC}(13)$.

In the present study, through the combined analysis of genome-wide profiles of $G 9 A$ obtained through chromatin immunoprecipitation-DNA sequencing (ChIP-Seq) and the gene expression dataset [from the Gene Expression Omnibus (GEO)] following $G 9 A-\mathrm{KO}$ in $\mathrm{BC}$, potential biomarkers were identified for the treatment of $\mathrm{BC}$. miRNAs that may regulate those biomarkers were screened out and the core miRNAs and genes were obtained. The findings of the present study may be valuable for the diagnosis and treatment of $\mathrm{BC}$.

\section{Materials and methods}

ChIP-Seq and gene expression dataset. In the present study, the ChIP-Seq and gene expression datasets were comprehensively obtained from the GEO database (http://www. ncbi.nlm.nih.gov/geo). The ChIP-Seq dataset (GSM1642516) was deposited from the study by Si et al (10), which used the G9A-specific antibody to capture the DNA fragments in MCF-7 cells and sequenced those fragments based on the GPL11154 Illumina HiSeq 2000 (Homo sapiens). Furthermore, an input control sample, which extracted all DNA in the human breast adenocarcinoma MCF-7 cell line, was adopted for the background correction in the identification of $G 9 A$ binding sites (peaks). The gene expression dataset GSE34925 (14) contained 3 G9A-KO and 3 control BC samples, which were based on GPL6244 (HuGene-1_0-st) Affymetrix Human Gene 1.0 ST Array [transcript (gene) version] (Affymetrix, Inc., Santa Clara, CA, USA).

Differential expression analysis. The raw CEL data (data storage format) were imported to $\mathrm{R}$ based on the affy package (15). Background correction and expression normalization was conducted using the oligo package (16). Probe IDs were converted to gene symbols via the annotation package of the microarray platform and the expression values were summarized that corresponding to multiple probe sets. A paired Student's t-test was conducted between logarithmic transformed expression values of G9A-KO samples and control samples. Differentially-expressed genes (DEGs) with a fold-change expression value of $>1.41$ or $<0.71$, and a false discovery rate corrected $\mathrm{P}$-value of $<0.05$ were screened out.
Functional enrichment analysis. The Database for Annotation, Visualization and Integrated Discovery (https://david.ncifcrf.gov/) (17) was used for the functional analysis of the DEGs. Gene Ontology (GO) terms and Kyoto Encyclopedia of Genes and Genomes (KEGG; http://www.kegg.jp/) pathways that satisfied the threshold of $\mathrm{P}<0.05$ were screened out.

Genome-wide binding analysis of G9A. Bowtie 2 (18), an ultrafast genome aligning tool, was used for the mapping of the raw reads to UCSC hg19 genome with a maximum of 2 mismatches in every read. Model-based analysis of ChIP-Seq version 14 (macs14) (19) was used for the identification of the binding sites of $G 9 A$ (also known as call peak) with a P-value of $<1 \times 10^{-5}$. Peaks located in -3000 to $3000 \mathrm{bp}$ centered in the transcription start sites (TSS) were considered to be conpeaks. Based on the ChIPseeker package (20), the nearby genes, genome features (including promoter and 5'-untranslated region) and distances to the nearest TSS were assigned to the peaks.

Construction of miRNA-gene network. The overlapping genes between DEGs and genes located in the conpeaks were screened out. miRNAs that may regulate those overlaps were identified using the TargetScan database (http://www. targetscan.org/) (21). The miRNA-gene regulation network was visualized through Cytoscape (http://www.cytoscape. $\operatorname{org} /)$.

\section{Results}

DEGs. A total of 217 DEGs were identified in the G9A-KO BC samples, and the distribution of these DEGs is demonstrated in Fig. 1. The supervised clustering (Fig. 2) based on those DEGs was able to separate the $G 9 A-\mathrm{KO}$ and control samples well.

Enriched functions. A total of 172 GO terms and 3 KEGG pathways were identified to be enriched in DEGs, and were mainly involved in the cell process, the inflammatory response and cancer progression, for example, the extracellular region part. The top $10 \mathrm{GO}$ terms according to P-value and KEGG pathways are presented in Tables I and II, respectively.

Genome-wide binding profile of G9A. A total of 10,422 binding sites (peaks) of $G 9 A$ were identified, including 1,210 conpeaks located in -3000 to 3000 bp centered in the TSS. The distribution of all peaks across the genome is shown in Fig. 3. In addition, 1,138 genes were identified to be located in the 1,210 conpeaks and 15 of these genes overlapped with DEGs (Table III).

miRNA-gene regulation network. Based on TargetScan, a total of 35 miRNAs were identified to regulate the 15 overlapping genes, and 65 miRNA-gene pairs were obtained among them. The miRNA-gene regulation network is shown in Fig. 4. The top 10 genes according to their connectivity in the miRNA-gene network are listed in Table IV. In the network, insulin-induced gene 1 (INSIGl) was regulated by 9 miRNAs and had the highest connectivity, which indicated that it was a hub gene. Furthermore, two other public 
Table I. Top 10 GO terms according to the P-value.

\begin{tabular}{llrl}
\hline Category & \multicolumn{1}{c}{ GO term } & Count & P-value \\
\hline CC & Extracellular space & 27 & $5.22 \times 10^{-8}$ \\
CC & Extracellular region part & 32 & $9.50 \times 10^{-8}$ \\
BP & Organ development & 44 & $1.04 \times 10^{-6}$ \\
BP & Anatomical structure & 53 & $1.44 \times 10^{-5}$ \\
& development & & \\
BP & System development & 50 & $1.59 \times 10^{-5}$ \\
BP & Integrin-mediated & 8 & $1.70 \times 10^{-5}$ \\
& signaling pathway & & \\
CC & Extracellular region & 44 & $1.95 \times 10^{-5}$ \\
BP & Inflammatory response & 15 & $2.81 \times 10^{-5}$ \\
BP & Blood vessel development & 13 & $3.09 \times 10^{-5}$ \\
BP & Defense response & 21 & $3.32 \times 10^{-5}$ \\
& & & \\
\hline
\end{tabular}

$\mathrm{GO}$, gene ontology; CC, cellular component; BP, biological process.

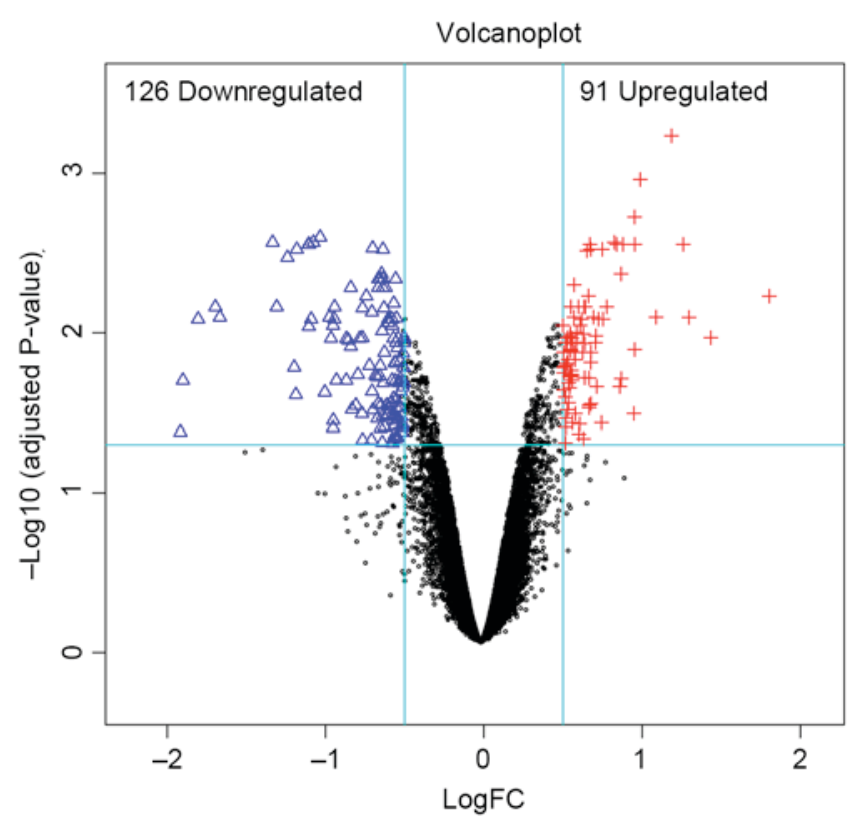

Figure 1.Distribution of DEGs in euchromatic histone lysine methyltransferase 2-knockout samples. The red plus signs and blue triangles represent upregulated and downregulated genes, respectively. The black circles represent non DEGs. FC, fold-change.

datasets (GSE29044 and GSE36774) were downloaded from the GEO database, which including data from breast cancer and normal tissue samples. These two datasets were background corrected and quantile normalized using the preprocessCore package of $\mathrm{R}$ (http://www.bioconductor. org/packages/release/bioc/html/preprocessCore.html). The Pearson and Spearman's correlation indices were respectively calculated using R between relative INSIG1 and G9A mRNA levels. The results indicated a significantly positive correlation (Fig. 5) between G9A and INSIG1, which may demonstrate their important roles in the progression of BC. The top 10 genes according to their connectivity in miRNA-gene network are presented in Fig. 4.

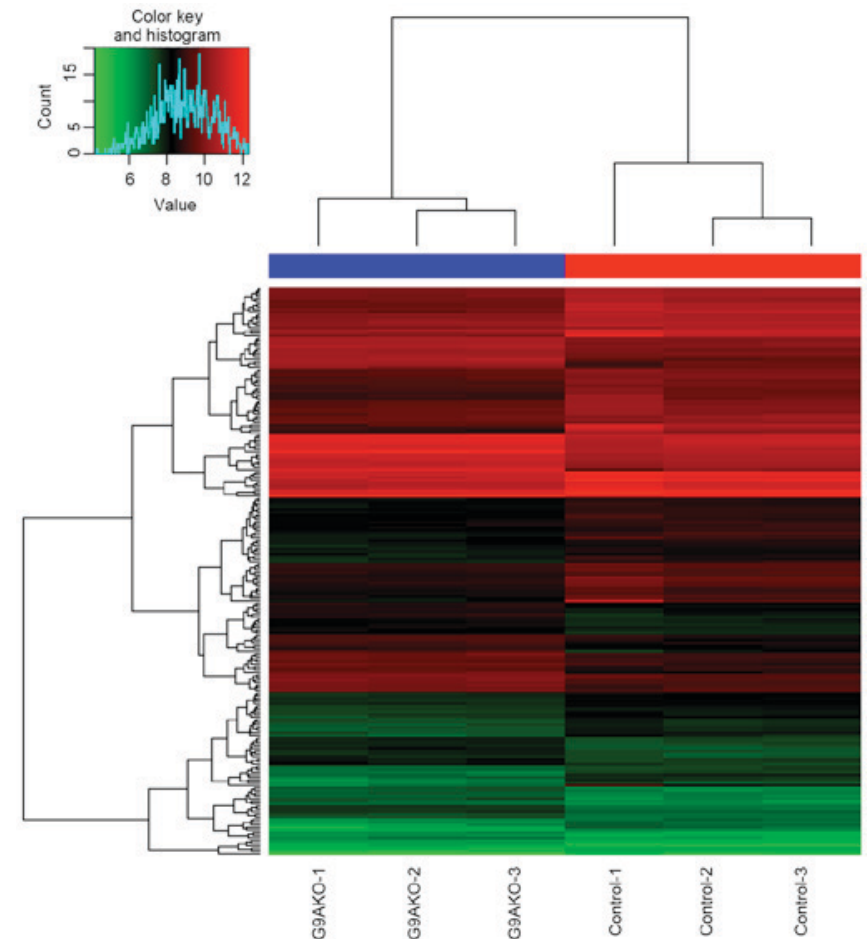

Figure 2. Two-way supervised clustering of differentially-expressed genes and samples using R software. $G 9 A$, euchromatic histone lysine methyltransferase $2 ; \mathrm{KO}$, knockout.

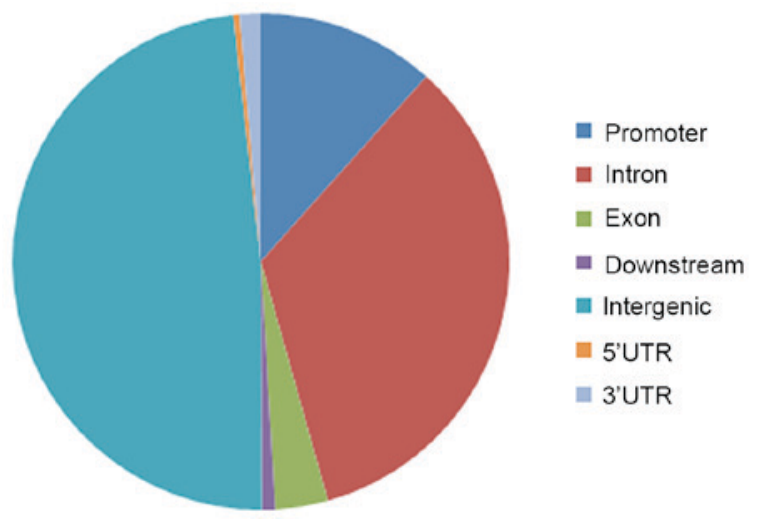

Figure 3. Distribution of peaks of euchromatic histone lysine methyltransferase 2 across the genome. UTR, untranslated region.

\section{Discussion}

$\mathrm{BC}$ is a complicated disease, and numerous factors may contribute to its initiation and progression, including occupational exposure (22), variations in expression level or mutations of specific genes $(23,24)$. The traditional treatment options for BC mainly involve surgical resection, chemotherapy and radiotherapy, which have been shown to be effective but rarely eradicate the disease. With the development of next-generation sequencing technology, numerous biomarkers of $\mathrm{BC}$ have been identified and certain novel therapeutics have been proposed (25). Immunotherapy is one of the most popular therapeutic treatment options $(26,27)$. The identification of novel biomarkers may therefore improve the prognosis for patients with BC. 
Table II. Enriched Kyoto Encyclopedia of Genes and Genomes pathways.

\begin{tabular}{|c|c|c|c|}
\hline Pathway name & Count & P-value & Genes \\
\hline Cytokine-cytokine receptor interaction & 13 & $2.99 \times 10^{-4}$ & $\begin{array}{l}C S F 2, T N F S F 4, I L 24, I L 7 R, C X C L 11, T N F S F 18, C X C L 10, \\
K D R, I N H B B, V E G F C, C X C L 16, I L 1 A, G H R\end{array}$ \\
\hline Focal adhesion & 11 & $5.24 \times 10^{-4}$ & $\begin{array}{l}\text { VEGFC, ITGB8, LAMA5, ITGB4, ITGA2, RELN, BIRC3, } \\
V A V 1, M Y L K, K D R, S P P 1\end{array}$ \\
\hline ECM-receptor interaction & 6 & $6.80 \times 10^{-3}$ & ITGB8, LAMA5, ITGB4, ITGA2, RELN, SPP1 \\
\hline
\end{tabular}

ECM, extracellular matrix.

Table III. Overlapping genes between differentially-expressed genes and genes located in conpeaks.

\begin{tabular}{lr}
\hline Gene & $\operatorname{logFC}$ \\
\hline SLCO4C1 & -0.65617 \\
ARHGAP24 & -0.66706 \\
ANK2 & -0.55143 \\
TMX4 & 0.54535 \\
LARP4 & -0.50197 \\
TC2N & -0.78340 \\
TMTC1 & 0.52521 \\
SLFN5 & -0.62773 \\
CYTIP & 0.67537 \\
PLCB4 & -0.55811 \\
ATF7IP2 & -1.19761 \\
CAMK2D & -0.50487 \\
TLR4 & 0.85905 \\
INSIG1 & -1.18712 \\
GHR & -0.60367 \\
\hline
\end{tabular}

FC, fold-change.

Table IV. Top 10 genes according to their connectivity in the microRNA-gene network.

\begin{tabular}{lc}
\hline Gene & Connectivity \\
\hline INSIG1 & 9 \\
ARHGAP24 & 8 \\
CAMK2D & 8 \\
GHR & 8 \\
ANK2 & 7 \\
TLR4 & 7 \\
TMX4 & 6 \\
CYTIP & 6 \\
miR-141 & 4 \\
miR-145 & 4 \\
\hline
\end{tabular}

In the present study, the genome-wide profiles of $G 9 A$ in genome wide were identified using ChIP-Seq. DEGs in

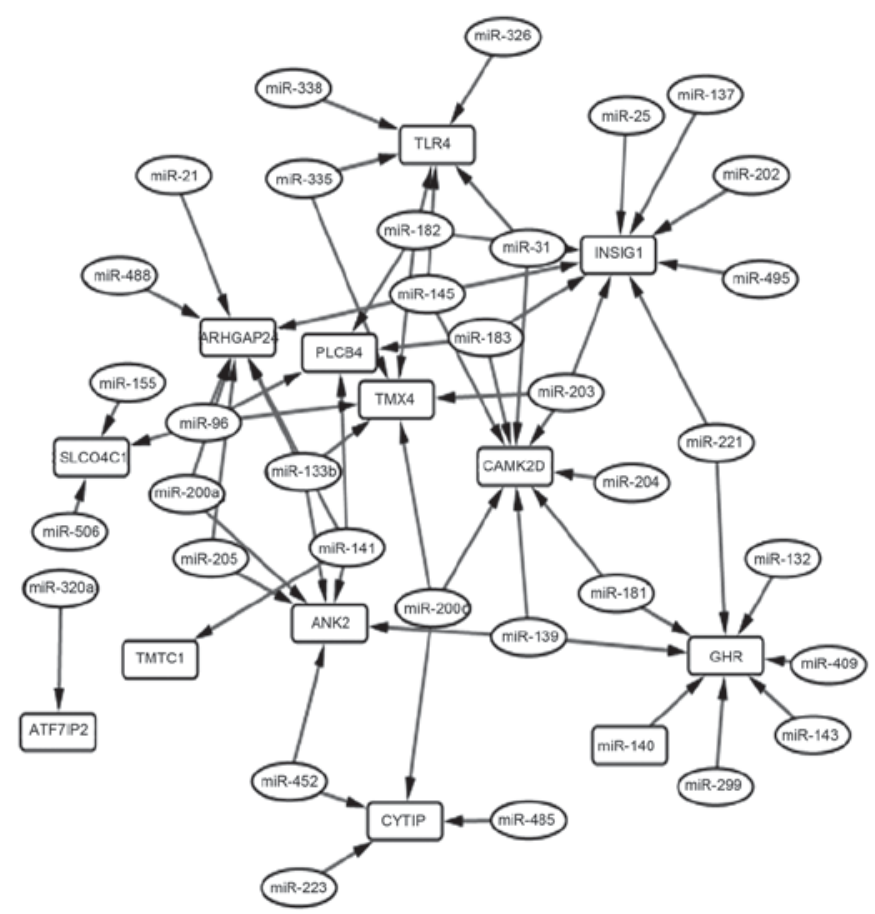

Figure 4. miRNA-gene regulation network. Rectangles show genes, whilst miRNAs are shown in ovals. Arrows represent the interactions between these genes and miRNAs.

G9A-KO BC samples compared with the control samples were screened out. The combined analysis obtained potential direct targets of $G 9 A$, the majority of which were downregulated in the $G 9 A-\mathrm{KO} \mathrm{BC}$ samples, which may indicate its upregulated functions in $\mathrm{BC}$.

DEGs in G9A-KO samples are primarily involved in biological processes or pathways associated with cellular processes, inflammatory responses and cancer progression, including extracellular region part, cytokine-cytokine receptor interaction and extracellular membrane (ECM) receptor interaction, all of which are closely associated with the development of BC. In particular, inflammatory triple-negative $\mathrm{BC}$, a type of rare and aggressive $\mathrm{BC}$ with a significantly poorer 5-year survival rate compared with other types of BC, is believed to mainly be attributed to an abnormal inflammatory response $(28,29)$. Previous studies have considered that the ECM-receptor interaction pathway may be associated with the progression of $\mathrm{BC}$, which could indicate the repeatability of the present study $(30,31)$. 
A

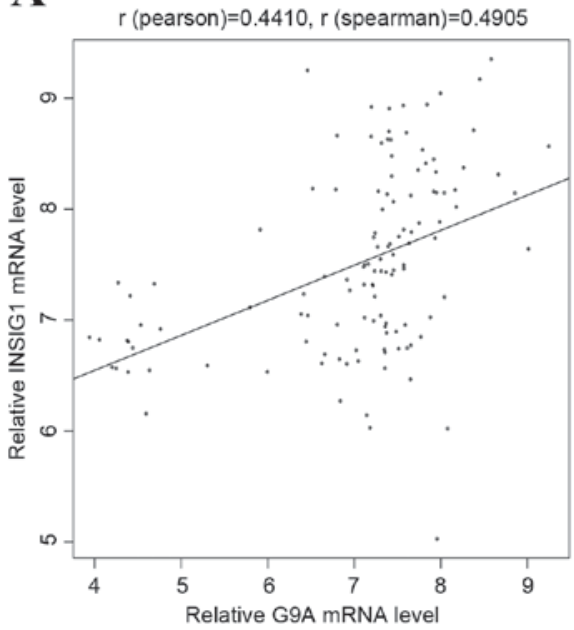

B

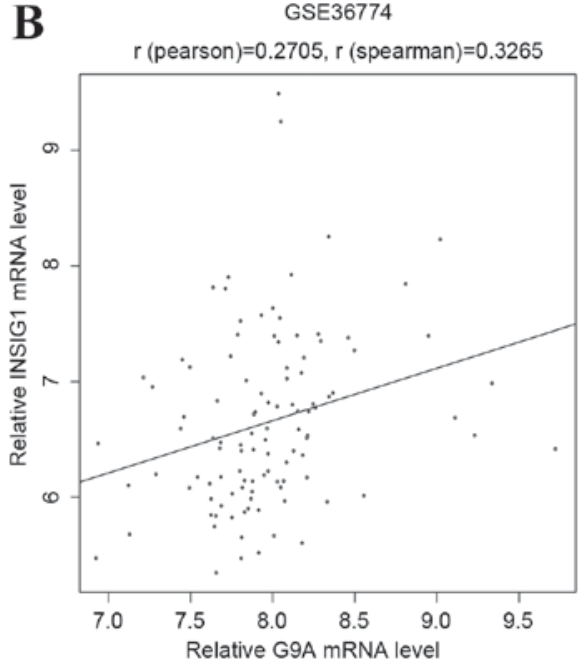

Figure 5. (A and B) Expression values of G9A were plotted against that of INSIG1 based on the other two public datasets (GSE29044 and GSE36774). G9A, euchromatic histone lysine methyltransferase 2; INSIG1, insulin-induced gene 1.

Among the 1,138 genes distributed in the 1,210 peaks located between -3000 to 3000 bp centered in the TSS, 15 were identified to overlapped with the DEGs in the G9A-KO $\mathrm{BC}$ samples. Furthermore, 11 of those overlaps were revealed to be downregulated in $G 9 A-\mathrm{KO}$ samples compared with the control samples, which may indicate that $G 9 A$ is mainly involved in the upregulation of its target genes. The positive correlation between the expression values of G9A and INSIGI was verified using two public datasets (Fig. 5). Furthermore, INSIG1 possessed an intimate connectivity pattern in the miRNA-gene regulation network (Fig. 4). Thus, this suggests that $G 9 A$ and INSIGI serve important roles in the progression of BC. INSIGI, which is downregulated in G9A-KO $\mathrm{BC}$, was closely associated with the progression of several types of cancer via the regulation of glucose metabolism and hypoxia-induced EMT $(32,33)$. BC had a strong association with insulin resistance, which was mediated by INSIG1 (34). In previous studies, INSIGI was also demonstrated to be differentially expressed in $\mathrm{BC}(35,36)$. Combined with the results of the present study, this indicates the important roles of INSIGI in BC.

miR-203 regulated 3 genes in the miRNA-gene regulation network, including INSIGI, which has been reported as a tumor suppressor in numerous types of cancer, including BC (37-39). The activity of $m i R-203$ was also affected by a number of other factors, including kallistatin (40). Therefore, it would be useful to study the upstream and downstream regulation loops of $m i R-203$ for the diagnosis and treatment of $\mathrm{BC}$, as well as other types of cancer. Other nodes (miRNAs or genes) with high connectivity in the miRNA-gene regulation network may also be potential biomarkers for $\mathrm{BC}$, which should be additionally verified through molecular biological experiments.

In conclusion, the present study conducted the combined analysis of ChIP-Seq and gene expression profiles in BC, and identified potential biomarkers via screening. The present results may aid the development of novel diagnostic or treatment methods, including immunotherapy, and therefore improve the prognosis of $\mathrm{BC}$.

\section{Acknowledgements}

The present study was supported by the Municipal Science and Technology Commission of Tianjin (grant nos. 15ZLZLZF00440 and 16ZLZXZF00120) and the Health Bureau Science and Technology Foundation of Tianjin (grant no. 2014KZ102).

\section{References}

1. Zhu J, Chen JG, Chen YS, Zhang YH, Ding LL and Chen TY: Female breast cancer survival in Qidong, China, 1972-2011: A population-based study. BMC Cancer 14: 318, 2014.

2. Fan L, Goss PE and Strasser-Weippl K: Current status and future projections of breast cancer in Asia. Breast Care (Basel) 10: 372-378, 2015.

3. Onega T, Tosteson AN, Weiss J, Alford-Teaster J, Hubbard RA, Henderson LM, Kerlikowske K, Goodrich ME, O'Donoghue C, Wernli KJ, et al: Costs of diagnostic and preoperative workup with and without breast MRI in older women with a breast cancer diagnosis. BMC Health Serv Res 16: 76, 2016.

4. Dare AJ, Anderson BO, Sullivan R, Pramesh CS, Andre I, Adewole IF, Badwe RA and Gauvreau CL: Surgical services for cancer care. 2015.

5. Daroudi R, Akbari Sari A, Nahvijou A, Kalaghchi B, Najafi M and Zendehdel K: The economic burden of breast cancer in Iran. Iran J Public Health 44: 1225-1233, 2015.

6. Zhang J, He P, Xi Y, Geng M, Chen Y and Ding J: Down-regulation of G9a triggers DNA damage response and inhibits colorectal cancer cells proliferation. Oncotarget 6: 2917-2927, 2015.

7. Liu S, Ye D, Guo W, Yu W, He Y, Hu J, Wang Y, Zhang L, Liao Y, Song H, et al: G9a is essential for EMT-mediated metastasis and maintenance of cancer stem cell-like characters in head and neck squamous cell carcinoma. Oncotarget 6: 6887-6901, 2015.

8. Laumet G, Garriga J, Chen SR, Zhang Y, Li DP, Smith TM, Dong Y, Jelinek J, Cesaroni M, Issa JP and Pan HL: G9a is essential for epigenetic silencing of $\left.\mathrm{K}^{+}\right)$channel genes in acute-to-chronic pain transition. Nat Neurosci 18: 1746-1755, 2015.

9. Lee JY, Lee SH, Heo SH, Kim KS, Kim C, Kim DK, Ko JJ and Park KS: Novel function of lysine methyltransferase G9a in the regulation of Sox2 protein stability. PLoS One 10: e0141118, 2015.

10. Si W, Huang W, Zheng Y, Yang Y, Liu X, Shan L, Zhou X, Wang Y, Su D, Gao J, et al: Dysfunction of the reciprocal feedback loop between GATA3- and ZEB2-nucleated repression programs contributes to breast cancer metastasis. Cancer Cell 27: 822-836, 2015. 
11. Liu X, Liao S, Xu Z, Zhu L, Yang F and Guo W: Identification and analysis of the porcine MicroRNA in porcine cytomegalovirus-infected macrophages using deep sequencing. PLoS One 11: e0150971, 2016.

12. Hu JY, Yi W, Zhang MY, Xu R, Zeng LS, Long XR, Zhou XM, Zheng XS, Kang Y and Wang HY: MicroRNA-711 is a prognostic factor for poor overall survival and has an oncogenic role in breast cancer. Oncol Lett 11: 2155-2163, 2016.

13. Ali HO, Arroyo AB, González-Conejero R, Stavik B, Iversen N, Sandset PM, Martínez C and Skretting G: The role of microRNA $-27 \mathrm{a} / \mathrm{b}$ and microRNA-494 in oestrogen mediated downregulation of tissue factor pathway inhibitor $\alpha$. J Thromb Haemost 14: 1226-1237, 2016.

14. Dong C, Wu Y, Yao J, Wang Y, Yu Y, Rychahou PG, Evers BM and Zhou BP: G9a interacts with Snail and is critical for Snail-mediated E-cadherin repression in human breast cancer. J Clin Invest 122 1469-1486, 2012

15. Gautier L, Cope L, Bolstad BM and Irizarry RA: affy-analysis of Affymetrix GeneChip data at the probe level. Bioinformatics 20: 307-315, 2004

16. Stuhlmüller B: A composition of nucleic acid sequences, specific for inflammatory disease, in particular rheumatoid arthritis. EP 1795610 A1. Filed December 6, 2005; issued June 13, 2007.

17. Dennis G Jr, Sherman BT, Hosack DA, Yang J, Gao W, Lane HC and Lempicki RA: DAVID: Database for annotation, visualization, and integrated discovery. Genome Biol 4: P3, 2003.

18. Langmead B and Salzberg SL: Fast gapped-read alignment with Bowtie 2. Nat Methods 9: 357-359, 2012.

19. Feng J, Liu T, Qin B, Zhang Y and Liu XS: Identifying ChIP-seq enrichment using MACS. Nat Protoc 7: 1728-1740, 2012

20. Yu G, Wang LG and He QY: ChIPseeker: An R/Bioconductor package for ChIP peak annotation, comparison and visualization. Bioinformatics 31: 2382-2383, 2015.

21. Song X, Cheng L, Zhou T, Guo X, Zhang X, Chen YP, Han P and Sha J: Predicting miRNA-mediated gene silencing mode based on miRNA-target duplex features. Comput Biol Med 42: 1-7, 2012.

22. Fenga C: Occupational exposure and risk of breast cancer. Biomed Rep 4: 282-292, 2016.

23. Inoue $\mathrm{K}$ and Fry EA: Novel molecular markers for breast cancer. Biomark Cancer 8: 25-42, 2016.

24. Rizk SM, Shahin NN and Shaker OG: Association between SIRT1 gene polymorphisms and breast cancer in Egyptians. PLoS One 11: e0151901, 2016.

25. Shen $\mathrm{Y}$ and Cai T: Identifying predictive markers for personalized treatment selection. Biometrics 72: 1017-1025, 2016.

26. Becht E, de Reyniès A, Giraldo NA, Pilati C, Buttard B, Lacroix L, Selves J, Sautès-Fridman C, Laurent-Puig Pa and Fridman WH: Immune and stromal classification of colorectal cancer is associated with molecular subtypes and relevant for precision immunotherapy. Clin Cancer Res 22: 4057-4066, 2016.

27. Abou-Shousha S, Moaz M, Sheta M and Motawea MA: An approach to breast cancer immunotherapy: The apoptotic activity of recombinant anti-interleukin-6 monoclonal antibodies in intact tumor microenvironment of breast carcinoma. Scand J Immunol 83: 427-437, 2016.
28. Biswas T, Efird JT, Prasad S, James SE, Walker PR and Zagar TM: Inflammatory TNBC breast cancer: Demography and clinical outcome in a large cohort of patients with TNBC. Clin Breast Cancer 16: 212-216, 2016.

29. Suárez-Arroyo IJ, Rios-Fuller TJ, Feliz-Mosquea YR, Lacourt-Ventura M,Leal-Alviarez DJ, Maldonado-Martinez G, Cubano LA and Martínez-Montemayor MM: Ganoderma lucidum combined with the EGFR tyrosine kinase inhibitor, erlotinib synergize to reduce inflammatory breast cancer progression. J Cancer 7: 500-511, 2016.

30. Yang X, Jia M, Li Z, Lu S, Qi X, Zhao B, Wang X, Rong Y, Shi J, Zhang Z, et al: Bioinformatics analysis of aggressive behavior of breast cancer via an integrated gene regulatory network. J Cancer Res Ther 10: 1013-1018, 2014.

31. Sun Y, Yuan K, Zhang P, Ma R, Zhang QW and Tian XS: Crosstalk analysis of pathways in breast cancer using a network model based on overlapping differentially expressed genes. Exp Ther Med 10: 743-748, 2015.

32. Chen HF and Wu KJ: Epigenetics, TET proteins, and hypoxia in epithelial-mesenchymal transition and tumorigenesis. Biomedicine (Taipei) 6: 1, 2016.

33. Tsai YP, Chen HF, Chen SY, Cheng WC, Wang HW, Shen ZJ, Song C, Teng SC, He C and Wu KJ: TET1 regulates hypoxia-induced epithelial-mesenchymal transition by acting as a co-activator. Genome Biol 15: 513, 2014

34. Ghose A, Kundu R, Toumeh A, Hornbeck C and Mohamed I: A review of obesity, insulin resistance, and the role of exercise in breast cancer patients. Nutr Cancer 67: 197-202, 2015.

35. Hernández-Vargas H, Rodriguez-Pinilla SM, Julián-Tendero M, Sánchez-Rovira P, Cuevas C, Antón A, Ríos MJ, Palacios J and Moreno-Bueno G: Gene expression profiling of breast cancer cells in response to gemcitabine: NF-kappaB pathway activation as a potential mechanism of resistance. Breast Cancer Res Treat 102: 157-172, 2007.

36. Einbond LS, Su T, Wu HA, Friedman R, Wang X, Jiang B, Hagan T, Kennelly EJ, Kronenberg F and Weinstein IB: Gene expression analysis of the mechanisms whereby black cohosh inhibits human breast cancer cell growth. Anticancer Res 27: 697-712, 2007.

37. Shi Y, Tan YJ,Zeng DZ, Qian F and Yu PW: miR-203 suppression in gastric carcinoma promotes Slug-mediated cancer metastasis. Tumour Biol: Jul 21, 2015 (Epub ahead of print).

38. Taipaleenmäki H, Browne G, Akech J, Zustin J, van Wijnen AJ, Stein JL, Hesse E, Stein GS and Lian JB: Targeting of Runx2 by miR-135 and miR-203 impairs progression of breast cancer and metastatic bone disease. Cancer Res 75: 1433-1444, 2015.

39. Zhou Y, Hu HY, Meng W, Jiang L, Zhang X, Sha JJ, Lu Z and Yao Y: MEK inhibitor effective against proliferation in breast cancer cell. Tumour Biol 35: 9269-9279, 2014.

40. Li P, Guo Y, Bledsoe G, Yang Z, Chao L and Chao J: Kallistatin induces breast cancer cell apoptosis and autophagy by modulating Wnt signaling and microRNA synthesis. Exp Cell Res 340: 305-314, 2016. 